\title{
Insatisfacción corporal en estudiantes de medicina de la Universidad Peruana Cayetano Heredia medida con el Body Shape Questionnaire.
}

\author{
Body dissatisfaction among medical students of the Universidad Peruana Cayetano Heredia measured \\ with the Body Shape Questionnaire.
}

\author{
Ricardo Benel Huerta ${ }^{1}$, Silvia Campos Campos ${ }^{1}$, Lizardo Cruzado ${ }^{2}$.
}

\section{RESUMEN}

La bibliografía científica informa que la mayoría de las mujeres están insatisfechas con su apariencia. El interés hacia la insatisfacción corporal surge debido a que es un factor modificable, predisponente, perpetuante y pronóstico de los desórdenes alimentarios y otros trastornos. Objetivo: Determinar el porcentaje de insatisfacción corporal en estudiantes universitarias, así como su relación con otros factores como la edad, actividad física e índice de masa corporal. Materiales y métodos: Se hizo un estudio transversal descriptivo donde participaron 124 estudiantes universitarias de la escuela de Medicina de edades entre 19 y 27 años. Los instrumentos de evaluación utilizados fueron el cuestionario sobre insatisfacción corporal (BSQ), el cuestionario de actividad física (IPAQ), y el Índice de Masa Corporal (IMC). Resultados: Un 60.4\% de las universitarias presentó valores compatibles con insatisfacción corporal, además a medida que aumenta el IMC fue mayor la insatisfacción corporal en las jóvenes universitarias. Por otro lado, considerando que los resultados no fueron estadísticamente significativos, no podemos concluir asociación entre la insatisfacción corporal y la edad, así como con el nivel de actividad física. Conclusiones: El porcentaje de insatisfacción corporal en mujeres es similar al hallado en otros estudios. Además se encontró asociación positiva y significativa al asociar el IMC e insatisfacción corporal. El elevado porcentaje de insatisfacción corporal impone el desarrollo de estrategias de prevención en grupos más vulnerables al desarrollo de trastornos asociados.

PALABRAS CLAVE: imagen corporal, actitud, índice de masa corporal, actividad física.

\section{SUMMARY}

Scientific evidence suggests that most college women are dissatisfied with their appearance. The interest in body dissatisfaction arises because it is a modifiable, predisposing, perpetuating and prognostic factor of eating disorders and other disorders. Objective: To determine the percentage of body dissatisfaction in university students and its relationship with other factors such as age, physical activity and body mass index. Materials and methods: It is a transversal descriptive study, applied to a sample of 124 university students of medical school between 19 and 27 years. The instruments used were Body Shape Questionnaire (BSQ), Physical Activity Questionnaire (IPAQ), and Body Mass Index (BMI). Results: $60.4 \%$ of the students showed compatible values with body dissatisfaction, as well as BMI increased greater body dissatisfaction level in the students. Moreover, considering that the outcomes

\footnotetext{
${ }^{1}$ Médico Cirujano. Facultad de Medicina Alberto Hurtado. Universidad Peruana Cayetano Heredia.

${ }^{2}$ Psiquiatra. Facultad de Medicina Alberto Hurtado. Universidad Peruana Cayetano Heredia. Lima, Perú. Instituto

Nacional de Salud Mental Honorio Delgado - Hideyo Noguchi. Lima, Perú.
} 
were not statistically significant, we cannot conclude association between body dissatisfaction and age and with the level of physical activity. Conclusions: The percentage of body dissatisfaction in women is similar to the found in other studies. In addition, we found significant positive association between BMI and body dissatisfaction. The high percentage of body dissatisfaction requires the implementation of prevention strategies looking for healthy body measurements.

KEYWORDS: body image, attitude, body mass index, physical activity.

\section{INTRODUCCION}

Cada cultura a lo largo del tiempo ha tenido un estereotipo de imagen corporal. Actualmente la cultura occidental promueve "la belleza del cuerpo" como meta para conseguir el éxito social. Una consecuencia observable es que los jóvenes otorgan importancia desmesurada a su cuerpo y apariencia física (1).

La imagen corporal es definida como la actitud valorativa hacia la apariencia del propio cuerpo; y se evalúa mediante dos aspectos importantes: la posible alteración de la imagen corporal y la satisfacción corporal (2). No se ha logrado una definición adecuada para la insatisfacción corporal, pero algunos autores la definen como la inconformidad del sujeto con su cuerpo o con aquellas partes del mismo que más preocupan a los que padecen trastornos de conducta alimentaria (3); o también como una preocupación exagerada que produce malestar hacia algún defecto imaginario o extremado de la apariencia física (4).

En la bibliografía extranjera los porcentajes de insatisfacción corporal en estudiantes universitarias varían entre 66 y $72 \%(5,6)$. En nuestro medio, Flores (7) encontró que el 79,9\% de las estudiantes universitarias encuestadas cambiarían su aspecto físico si les fuera posible, casi en su totalidad por razones estéticas.

Adicionalmente, la insatisfacción corporal puede constituir un factor predisponente, perpetuante y pronóstico de desórdenes alimentarios y su persistencia constituye un riesgo de recaída $(8,9$, 10). Un meta-análisis concluyó que las anoréxicas y bulímicas poseen mayor insatisfacción corporal y distorsión perceptiva del cuerpo que las mujeres sin patologías alimentarias (11). También se conoce que la insatisfacción corporal es un factor de riesgo para depresión (12) y problemas de baja auto-estima (13).

Se cuenta con diversos instrumentos para evaluar la insatisfacción corporal, uno de los cuales es el Body Shape Questionnaire (BSQ) (14). Este test fue adaptado a la población española (4), colombiana
(15) y mexicana (16); y en nuestro país está validado y es un primer acercamiento a la investigación de la insatisfacción corporal en la juventud peruana. (7)

La evidencia señala al IMC como un indicador muy importante para predecir la satisfacción corporal (1). Se destaca que las mujeres con elevado IMC poseen mayor insatisfacción corporal $(1,18,19,20)$. Sin embargo, otras investigaciones contradicen estos resultados al no encontrar diferencias significativas que relacionen la insatisfacción corporal y el IMC $(17,21)$. Asimismo, algunos autores afirman que la actividad física se relaciona con mayor satisfacción corporal y autoestima más elevada (22), mientras que en otros estudios una imagen corporal negativa previa inducía a realizar ejercicio para modelar el cuerpo. En relación a la edad, algunas publicaciones reportan que las mujeres más afectadas suelen tener entre 19 y 23 años, así como las mujeres mayores de 60 años (23). Sin embargo, en estudios realizados en nuestro medio (6) se halló que no existe asociación entre la edad y el nivel de insatisfacción corporal. Considerando esta diversidad de enfoques, se hace necesario evaluar la relación entre los factores previamente descritos y la imagen corporal.

En nuestra población existen algunos estudios sobre insatisfacción corporal, pero la información es limitada e insuficiente. Por ello, el presente estudio busca mediante el cuestionario Body Shape Questionnaire, determinar el porcentaje de insatisfacción corporal en estudiantes universitarias de medicina humana de la Universidad Peruana Cayetano Heredia (UPCH) en el año 2012. Asimismo, pretendemos describir la relación entre insatisfacción corporal con otros factores como la edad, actividad física e IMC.

\section{MATERIALES Y METODOS}

\section{Participantes}

Un total de 124 estudiantes de Medicina de la $\mathrm{UPCH}$, mayores de 18 años de edad, participaron en este estudio transversal descriptivo. La muestra se eligió por conveniencia según la disponibilidad de tiempo de las estudiantes, y se incluyó a estudiantes de $4^{\circ}$ a $7^{\circ}$ años de la escuela de medicina. Se aplicaron 
138 encuestas, sin embargo se excluyeron 14 encuestas debido a que se encontraban mal llenadas o incompletas.

\section{Variables e instrumentos}

Insatisfacción corporal: Se midió con el BSQ, que comprende 34 preguntas sobre la imagen corporal y tiene una escala de frecuencia que va desde 1 punto que equivale a nunca, hasta 6 puntos que equivale a siempre, de modo que el rango de la prueba es de 34 a 204 (14). De acuerdo a la versión original del BSQ el punto de corte para insatisfacción corporal es mayor a 81, por lo cual se utilizó este valor para nuestro estudio. Este es un instrumento que ha sido validado en el Perú (7) para la evaluación de la insatisfacción corporal en la muestra de adolescentes universitarias, mostrando indicadores sólidos de validez convergente y de criterio por diferenciación entre grupos, así como alta confiabilidad por consistencia interna $(\alpha=0.97)$ y adecuada capacidad discriminativa de todos los ítems. Índice de masa corporal (IMC): Es un indicador del estado nutricional, y se calcula dividiendo el peso en kilos por el cuadrado de su talla en metros $\left(\mathrm{kg} / \mathrm{m}^{2}\right)$ Edad: Se medirá en años enteros cumplidos. Actividad física: Es todo movimiento del cuerpo que hace trabajar a los músculos y requiere más energía que estar en reposo. El Cuestionario Internacional de Actividad Física (IPAQ) está disponible públicamente y permite clasificar la intensidad de la actividad física. Este instrumento fue desarrollado por un grupo de expertos en un Consenso Internacional, en Ginebra en 1998, para facilitar la vigilancia de actividad física basada en un estándar global y continuó con ensayos extensivos de confiabilidad y validación llevados a cabo en 12 países durante el 2003 (17). Ha sido el cuestionario sobre actividad física más utilizado alrededor del mundo, con dos versiones disponibles, una extendida y otra abreviada. La actividad física está medida en "MET-minutos" que resultan de multiplicar el factor o puntaje MET de una actividad determinada por la cantidad de minutos que se practica esa actividad. Utilizando el puntaje METs, la frecuencia de la actividad física y su intensidad, se clasifica en categoría baja, moderada o alta, según el baremo del IPAQ.

\section{Procedimiento}

En diferentes momentos según disponibilidad de tiempo de los participantes, se ingresó a las aulas de las estudiantes desde cuarto a séptimo año de la escuela de medicina, con anuencia del docente y se explicó las bases del estudio. Luego se solicitó la presencia de sólo las estudiantes mujeres y se entregó los formatos a quienes decidieron participar en el estudio. En primer lugar se entregó el consentimiento informado y se solicitó que entreguen el desglose de la parte superior de la encuesta. Este desglose contenía el mismo código de los cuestionarios y nombre de la persona que llenó los cuestionarios previamente para conservar el anonimato. En segundo lugar realizaron el llenado de los cuestionarios BSQ e IPAQ. Finalmente, los investigadores utilizaron los desgloses con los nombres de las participantes para recolectar el IMC de la base de datos de la Clínica Cayetano Heredia.

\section{Plan de análisis}

Tras efectuar una depuración y filtrado de los datos introducidos haciendo uso del programa STATA versión 12 , se procedió a analizar la distribución de las mediciones. De acuerdo a la normalidad de las variables, se realizó los análisis de asociación entre dos variables, y se empleó coeficientes de correlación de Pearson, prueba $\mathrm{T}$ de Student, Anova y $\mathrm{Chi}^{2}$ según sea necesario. El nivel de significación que se utilizó para determinar la significancia estadística, fue $\alpha=0,05$. Asimismo se elaboraron tablas de doble entrada y gráficos tales como diagrama de puntos.

\section{RESULTADOS}

La muestra del estudio fue de 124 mujeres universitarias entre 19 a 27 años con un promedio de edad de 22,9 ( $\mathrm{DE}=1,48)$. Se dividió en dos grupos, el primero entre 19 a 23 años y otro entre 24 a 27 años, que correspondían al $64,5 \%$ y $35,4 \%$ respectivamente (Tabla No1).

Con respecto a las puntuaciones de BSQ, 75 mujeres $(60,5 \%)$ se encontraron insatisfechas con su cuerpo y $49(39,5 \%)$ no se encontraron insatisfechas (Tabla $\mathrm{N}^{\circ} 2$ ). Además, se aplicó la prueba $\mathrm{T}$ de student para muestras independientes, y previamente se corroboró el supuesto de normalidad para ambos grupos con la prueba de Kolmogorov Smirnov $(p>0,05)$. Se obtuvo que la puntuación media del BSQ del grupo insatisfecho fue 108,9 (DE= 24,5, IC al 95\% 103,2 - 114,5), y la media del grupo satisfecho fue de 63,1

\begin{tabular}{|c|c|c|}
\hline $\begin{array}{l}\text { Edad } \\
\text { (años) }\end{array}$ & $\begin{array}{l}\text { Frecuencia } \\
\text { (n) }\end{array}$ & $\begin{array}{c}\text { Porcentaje } \\
(\%)\end{array}$ \\
\hline $19-23$ & 80 & 64,52 \\
\hline $24-27$ & 44 & 35,48 \\
\hline Total & 124 & 100,00 \\
\hline
\end{tabular}


Tabla $\mathbf{N}^{\circ}$ 2.Porcentaje de insatisfacción corporal

\begin{tabular}{ccc}
\hline BSQ & Frecuencia & Porcentaje \\
\hline Satisfecho & 49 & 39,52 \\
Insatisfecho & 75 & 60,48 \\
Total & 124 & 100,00 \\
\hline
\end{tabular}

Tabla No3. Media de puntaje de BSQ

\begin{tabular}{ccc}
\hline & Media & DE \\
\hline Satisfechos & 63,06 & 12,09 \\
Insatisfechos & 108,88 & 24,49 \\
Total & 90,77 & 30,4 \\
\hline $\mathrm{t}=-13,83$ & $\mathrm{p}=0,000$ &
\end{tabular}

Tabla N ${ }^{\circ}$. Media de puntaje de BSQ por edad

\begin{tabular}{cccc}
\hline & \multicolumn{3}{c}{ Puntajes del BSQ } \\
Edad & Frecuencia & Media & [IC al 95\%] \\
\hline $19-23$ & 80 & 93,99 & $86,73-101,25$ \\
$24-27$ & 44 & 84,93 & $77,29-92,58$ \\
Total & 124 & 90,77 & \\
\hline $\mathrm{t}=1,59$ & $\mathrm{p}=0,11$ & &
\end{tabular}

Tabla $\mathbf{N}^{\circ} 5$. Porcentaje de insatisfacción corporal de acuerdo a edad

\begin{tabular}{ccccc}
\hline & \multicolumn{3}{c}{ BSQ } \\
& \multicolumn{2}{c}{ Satisfechas } & \multicolumn{2}{c}{ Insatisfechas } \\
\hline Edad & $\mathrm{f}$ & $\%$ & $\mathrm{f}$ & $\%$ \\
$19-23$ & 31 & 63,27 & 49 & 65,33 \\
$24-27$ & 18 & 36,73 & 26 & 34,67 \\
Total & 49 & 100,00 & 75 & 100,00 \\
\hline Chi2(1) $=0,0554$ & $\mathrm{p}=0,814$ & &
\end{tabular}

$(\mathrm{DE}=12,1, \mathrm{IC}$ al 95\% 59,6 - 66,6), con significancia estadística $(\mathrm{t}=-13,8, \mathrm{p}<0,05)$ (Tabla $\left.\mathrm{N}^{\mathrm{o}} 3\right)$. Tomando en cuenta los puntos de corte de la $\mu+-2 \sigma$, el grupo de "muy insatisfechas" correspondió al 14,5\% y el grupo de "nada o poco insatisfechas" correspondió al $15,3 \%$. Además, debemos apreciar que la media del puntaje del BSQ del grupo de mujeres más jóvenes fue 93,9 y para las mujeres entre 24 y 27 años fue 84,9 , y se observó que para el grupo más joven la media era mayor. Sin embargo, esta diferencia no fue estadísticamente significativa $(\mathrm{t}=1,6 ; \mathrm{p}>0,05)$ (Tabla $\mathrm{N}^{\circ} 4$ ). Asimismo, de aquellas estudiantes con puntajes altos de BSQ, es decir las jóvenes con insatisfacción corporal, el 65,3\% tenía entre 19 y 23 años y 34,6\% tenía entre 24 y 27 años. Pero esta asociación entre edad e insatisfacción corporal no fue estadísticamente significativa $\left[\mathrm{Chi}^{2}(1)=0,06, \mathrm{p}>0,05\right]\left(\right.$ Tabla $\left.\mathrm{N}^{0} 5\right)$.

Por otro lado, respecto a la asociación con actividad física, se observó que el valor promedio de los puntajes del BSQ para el grupo con actividad física leve fue de 89,7; para el grupo con actividad física moderada de 92 y para el grupo de actividad física intensa de 99,6. Al realizar el análisis de varianza (ANOVA) se encontró que la diferencia entre las medias de estos grupos no era estadísticamente significativa $(\mathrm{F}=$ $0,4 ; p>0,05)$ (Tabla $\mathrm{N}^{\mathrm{o}} 6$ ). De las estudiantes con un nivel de actividad física leve, el $58,5 \%$ presentó insatisfacción corporal, además el $68,2 \%$ de las estudiantes con un nivel moderado de actividad física tenían insatisfacción corporal, mientras que $62,5 \%$ de las estudiantes con un nivel intenso de actividad física presentó insatisfacción corporal (Tabla $\mathrm{N}^{0} 7$ ). Se apreció que al aumentar el nivel de actividad física de leve a moderado, el porcentaje de insatisfacción corporal también aumentó, pero en el nivel intenso no siguió el mismo patrón. Luego de realizar la prueba estadística $\mathrm{Chi}^{2}$ exacta de Fisher, obtuvimos que el valor de $\mathrm{p}$ era mayor a 0,05 ; por lo tanto no pudimos concluir asociación entre la insatisfacción corporal y el nivel de actividad física. $\left[\mathrm{Chi}^{2}(2)\right.$ exacta $=0,7$; $\mathrm{p}>0,05]$. Finalmente, los puntajes del BSQ e IMC presentaron correlación y se aplicó la prueba no paramétrica de Kruskal - Wallis, donde apreciamos que la media de rangos fue mayor en el grupo de obesos, luego en el grupo de sobrepeso, seguido del grupo de peso normal y finalmente del grupo de desnutridos. Por lo tanto pudimos observar una gran discrepancia de los puntajes entre el grupo de obesos y desnutridos. En conclusión, al menos entre dos grupos del estado nutricional hubieron diferencias de puntajes

Tabla No6. Puntaje de insatisfacción corporal en grupos de actividad física

\begin{tabular}{cccc}
\hline \multicolumn{4}{c}{ Puntajes del BSQ } \\
\hline IPAQ & Frecuencia & Media & DS \\
\hline Leve & 94 & 89,73 & 31,12 \\
Moderado & 22 & 92,00 & 24,68 \\
Intenso & 8 & 99,63 & 37,79 \\
Total & 124 & 99,77 & 30,39 \\
\hline $\mathrm{F}=0,41$ & $\mathrm{p}=0,666$
\end{tabular}


Tabla $\mathbf{N}^{0} 7$. Insatisfacción corporal en grupos de actividad física

\begin{tabular}{ccccccccc}
\hline & & \multicolumn{7}{c}{ IPAQ } \\
\cline { 2 - 8 } BSQ & $\mathrm{f}$ & $\%$ & $\mathrm{f}$ & $\%$ & $\mathrm{f}$ & $\%$ & $\mathrm{f}$ & $\%$ \\
\hline Satisfechas & 39 & 41,49 & 7 & 31,82 & 3 & 37,50 & 49 & 39,52 \\
Insatisfechas & 55 & 58,51 & 15 & 68,18 & 5 & 62,50 & 75 & 60,48 \\
Total & 94 & 100,00 & 22 & 100,00 & 8 & 100,00 & 124 & 100,00 \\
\hline
\end{tabular}

Chi2(2) exacta $=0,7122 \quad \mathrm{p}=0,72$

Tabla N ${ }^{\circ} 8$. Media de rangos de puntuaciones de BSQ de acuerdo a grupos de IMC.

\begin{tabular}{cccc}
\hline & \multicolumn{3}{c}{ Puntajes del BSQ } \\
IMC & Frecuencia & Rango suma & Media de Rangos \\
\hline Desnutrido & 4 & 79,00 & 19,75 \\
Normal & 100 & 6169,00 & 61,69 \\
Sobrepeso & 16 & 1199,00 & 74,94 \\
Obeso & 4 & 302,50 & 75,62 \\
Total & 124 & 7750,00 & 62,5 \\
\hline Chi2(3)=8,172 & $\mathrm{p}=0,0426$
\end{tabular}

Tabla Nº. Insatisfacción corporal de acuerdo a grupos de IMC.

\begin{tabular}{ccccccccccc}
\hline & \multicolumn{1}{c}{ Desnutrido } & \multicolumn{1}{c}{ Normal } & \multicolumn{2}{c}{ Sobrepeso } & \multicolumn{1}{c}{ Obeso } & \multicolumn{2}{c}{ Total } \\
\cline { 2 - 12 } & $\mathrm{f}$ & $\%$ & $\mathrm{f}$ & $\%$ & $\mathrm{f}$ & $\%$ & $\mathrm{f}$ & $\%$ & $\mathrm{f}$ & $\%$ \\
BSQ & 4 & 100,00 & 42 & 42,0 & 3 & 18,75 & 0 & 0,00 & 63 & 50,81 \\
Satisfechas & 0 & 0,00 & 58 & 58,0 & 13 & 81,25 & 4 & 100,00 & 61 & 49,19 \\
Insatisfechas & 0 & 100,00 & 100 & 100,00 & 16 & 100,00 & 4 & 100,00 & 124 & 100,00 \\
Total & 4 & 10 & & & & & & & &
\end{tabular}

del BSQ $\left(\mathrm{Chi}^{2}(3)=8,2 ; \mathrm{p}<0,05\right)$ (Tabla 8). Del mismo modo, se encontró que el $100 \%$ de las estudiantes desnutridas estaban satisfechas con su cuerpo, mientras que las estudiantes con estado nutricional normal, sobrepeso y obesidad presentó $58 \% ; 81,3 \%$ y $100 \%$ de insatisfacción corporal respectivamente (Tabla 9). Se realizó la prueba estadística de chi cuadrado exacta de Fisher y se encontró asociación entre la insatisfacción corporal y el nivel del IMC $\left(\mathrm{Chi}^{2}\right.$ (3) exacta $=11,9$; $\mathrm{p}<0,05)$. En conclusión, aquellas mujeres cuyo nivel de peso se encuentra por debajo del peso adecuado según el IMC reportado, se sienten más satisfechas con la imagen corporal que poseen, que aquellas que se encuentran con sobrepeso $u$ obesidad e incluso aquellas que presentan un peso normal.

\section{DISCUSIÓN}

En los últimos años la sobrevaloración de la imagen corporal se ha incrementado en el contexto de una cultura basada en la percepción y la importancia de una imagen idealizada (24). Esta tendencia es encabezada por los medios de comunicación y la presión social, y afecta sobre todo a mujeres muy preocupadas por su peso y cuerpo (25). En el presente estudio, el $60.4 \%$ de las jóvenes presentó valores compatibles con insatisfacción corporal según el cuestionario BSQ. Este valor es ligeramente inferior comparado con los encontrados en la bibliografía extranjera donde se encontró hasta $72,3 \%$ de universitarias insatisfechas con su imagen corporal (5). Asimismo, Arroyo y cols. (6) obtuvieron en su estudio un $66 \%$ de universitarias insatisfechas con su imagen corporal. En nuestro país, 
Flores (2009) halló en universitarias de las carreras de letras y arquitectura una media del BSQ del grupo de insatisfechas de 142,3 (DS=30,8), y la media del grupo satisfechas de 66,24 (DS= 23,08). Estas puntuaciones son superiores en comparación con las halladas en nuestro estudio donde la media de satisfechas es 63,1 $(\mathrm{DS}=12,1)$ y de insatisfechas de $108,9(\mathrm{DS}=24,5)$.

En relación a la edad, las comparaciones realizadas mostraron que las jóvenes entre 19 y 23 años presentaron mayor insatisfacción frente a las de 24 a 27 años, sin embargo estas diferencias no fueron estadísticamente significativas, lo que coincide con una revisión similar que no demostró asociación entre estas variables (7). Empero, se debe considerar que nuestro estudio considera un pequeño rango de edad (19-27 años), lo cual no podría permitir una evaluación adecuada. Al analizar otras investigaciones $(26,27)$, los rangos de edad son más amplios, incluso a través de diversas etapas de la vida. No obstante, un estudio comparó la insatisfacción corporal a través de la edad e identificó mayor grado de insatisfacción entre los más jóvenes, de 19 a 23 años; así como en mujeres de edad avanzada. (23). Por otro lado, una revisión apunta hacia un grupo especialmente vulnerable, con un alto grado insatisfacción corporal, de mujeres entre 35 a 44 años. (29). En contraste, otros refieren que la insatisfacción corporal se mantiene estable durante toda la vida adulta de las mujeres o al menos hasta que son bastante mayores $(26,28)$.

En el presente estudio observamos una aparente relación entre aumento de actividad física e insatisfacción corporal, sin embargo, la diferencia entre las medias de estos grupos no es estadísticamente significativa. Asimismo, al evaluar la frecuencia de universitarias insatisfechas según la intensidad de actividad física, no se encontró significación estadística ni una relación directa puesto que el mayor porcentaje fue para el grupo de intensidad moderada (68\%) comparado con $62,5 \%$ del grupo que realizaba actividad física intensa. En conclusión, en nuestro estudio no podemos concluir asociación entre la insatisfacción corporal y el nivel de actividad física. De acuerdo a estudios previos, la actividad física se relaciona con una imagen corporal positiva y mayor satisfacción con su apariencia física $(22,29,30)$. De este modo, la participación deportiva se asocia a una autoestima más elevada, que protegería contra los trastornos de la imagen corporal y los desórdenes alimentarios (31, 32). En contraste otras investigaciones apuntan hacia un efecto potencialmente negativo del ejercicio sobre la imagen corporal $(33,34)$.
Respecto a la relación entre el IMC y el BSQ, se encontró una asociación positiva y significativa al asociar el IMC e insatisfacción corporal, debido a que en la medida en que aumenta el IMC se incrementa la insatisfacción corporal en las jóvenes, lo que es congruente con otros estudios realizados $(1,19)$, donde se reporta que las mujeres más satisfechas son las que poseen IMC más bajos (35). En contraposición, nuestros datos no coinciden con los obtenidos por otros investigadores, quienes no encuentran relación alguna entre dichas variables $(17,21)$. Asímismo, se identificó que las mujeres que tenían incluso un IMC saludable tenían alto porcentaje de insatisfacción corporal (58\%). Este hallazgo es explicable por el hecho de la aspiración de la forma del cuerpo ideal está muy sesgado por las normas socioculturales y los medios de comunicación $(36,37)$.

La insatisfacción corporal es un factor de riesgo para trastornos de conductas alimentarias (38), depresión $(12,38)$ y baja auto-estima (13), por lo tanto, es importante prevenir o tratar la insatisfacción corporal. En esta investigación, al analizar los factores relacionados con la insatisfacción, una de las acciones educativas en las mujeres podría ser buscar medidas corporales saludables, modificando el IMC.

De este trabajo se derivan posibles líneas de investigación: confirmar la validez del BSQ estableciendo un punto de corte de acuerdo a una población sana y enferma, análogamente a estudios similares en diversas partes del mundo $(14,15$, 16); comprobar si los programas de prevención de insatisfacción corporal en las mujeres jóvenes pueden reducir los valores obtenidos en esta investigación y por último, observar la relación existente entre puntuaciones altas de insatisfacción corporal y riesgo de sufrir un trastorno de conducta alimentaria en nuestra población.

En conclusión, se determinó que la mayoría de las estudiantes evaluadas presentó insatisfacción corporal $(60,4 \%)$ y además no se encontró asociación entre insatisfacción corporal y las variables de actividad física ni con la edad. En cambio, según nuestros resultados sí hubo una asociación entre IMC e insatisfacción corporal. Sin embargo, los estudios en nuestro medio que tratan de esclarecer si existen relaciones entre la satisfacción corporal y las variables planteadas, son escasos y por ello se deberían desarrollar nuevas investigaciones para establecer conceptos finales. 


\section{Correspondencia:}

Silvia Campos Campos.

Calle Monge 105. Dpto. 302. San Borja.

Lima, Perú. Lima 41.

Correo electrónico: silvia.campos@upch.pe

\section{Conflictos de interés:}

Los autores declaran no tener conflictos por declarar.

\section{REFERENCIAS BIBLIOGRÁFICAS}

1. Martínez D, Veiga O. Insatisfacción corporal en adolescentes: relaciones con la actividad física e índice de masa corporal. Revista internacional de medicina y ciencias de la actividad física y del deporte. 2007; 7: 253-265.

2. Bruchon-Schweitzar M. Psicología del Cuerpo. Barcelona: Herder; 1992.

3. Garner DM. Inventario de Trastornos de la Conducta Alimentaria (EDI-2)-Manual. Madrid: TEA; 1998.

4. Raich RM, Mora M, Soler A, Clos I, Zapater L. Adaptación de un instrumento de evaluación de la insatisfacción corporal. Clínica y salud. 1996; 7: 51-66.

5. Amaral D, Hernández N, Basabe N, Rocandio A, Arroyo M. Satisfacción corporal y calidad de la dieta en estudiantes universitarias del país vasco. Endocrinol Nutr. 2012; 59: 239-245.

6. Arroyo M, Rocandio P, Ansotegui A. Imagen corporal en estudiantes universitarios: comportamientos dietéticos y actitudes nutricionales. Osasunaz. Cuadernos de Ciencias de la salud. 2007; 8: 199-215.

7. Flores M. Validez y confiabilidad del Body Shape Questionnaire en adolescentes universitarias de Lima Metropolitana. Tesis de bachiller. Biblioteca de la Pontificia Universidad Católica del Perú; 2009.

8. Behar R, Alviña M, González T, Rivera N. Detección de actitudes y/o conductas predisponentes a trastornos alimentarios en estudiantes de enseñanza media de tres colegios particulares de Viña del Mar. Rev Chil Nut. 2007; 34: 1-25.

9. Fernández $\mathrm{M}$, Otero $\mathrm{M}$, Rodríguez $\mathrm{Y}$, Fernández Prieto M. Hábitos alimentarios e imagen corporal en estudiantes universitarios sin trastornos alimentarios. Int J Clin Health Psych. 2003; 1 (3): 23-33.

10. Rivarola M. La imagen corporal en adolescentes mujeres: $\mathrm{Su}$ valor predictivo en trastornos alimentarios. Fundamentos en Humanidades. 2003; 4 (I-II): 149-162.

11. Cash T, Deagle E. The nature and extent of body image disturbance in anorexia nervosa and bulimia nervosa: A meta-analysis. Int J Eat Dis. 1997; 22: 107-125.

12. Brausch AM, Gutiérrez PM. The role of body image and disordered eating as risk factors for depression and suicidal ideation in adolescents. Suicide Life Threat Behav. 2009 ; 39: 58-71.

13. Paxton SJ, Neumark-Sztainer D, Hannon PJ, Eisenberg ME. Body dissatisfaction prospectively predicts depressed mood and low self-esteem in girls and boys. J Clinic Child Adolescent Psychol. 2006; 35: 539-549.

14. Cooper J, Taylor M, Cooper Z, Fairburn C. The development and validation of the body shape questionnaire. Int J Eat Disord. 1987; 6:485-494.

15. Castrillón D, Luna I, Avendaño G. Validation of the body shape questionnaire for Colombian population. Acta colombiana de psicología. 2007; 10(1):15-23.

16. Vázquez R, Galán J, López X, Álvarez G, Mancilla J, Caballero R, et al. Validez del Body Shape Questionnaire (BSQ) en Mujeres Mexicanas. Revista Mexicana de Trastornos Alimentarios. 2011; 2, 42520.

17. Jones D. Body image among adolescent girls and boys: a longitudinal study. Dev Psicol. 2004; 40: 823835.

18. Craig CL, Marshall AL, Sjostrom M, Bauman A, Booth ML, Ainsworth BE, Pratt M, Ekelund U, Yngve A, Sallis JF, Oja P: International Physical Activity Questionnaire: 12-country reliability and validity. Med Sci Sports Exerc. 2003; 35: 1381-1395.

19. Cruz S, Maganto M. Índice de masa corporal, preocupación por la delgadez y satisfacción corporal en adolescentes. Rev Psicol Gral Aplic. 2002; 55: 455-473.

20. Rodríguez, S. y Cruz, S. Insatisfacción corporal en adolescentes latinoamericanas y españolas. Psicothema. 2008; 20: 131-137.

21. Stice E, Shaw HE. Role of body dissatisfaction in the onset and maintenance of eating pathology: A synthesis of research findings. J Psychosom Res. 2002; 53: 985-993.

22. Ballester F, Gracia M, Patiño J. Actitudes alimentarias y satisfacción corporal en adolescentes: un estudio de prevalencia. Actas Esp Psiquiatr. 2002; 30: 207-312

23. Waaler N. Body image and physical activity: A survey among Norwegian men and women. Int J Sports Psychol. 1998; 29: 339-365.

24. Bedford J, Johnson C. Societal Influences on Body Image Dissatisfaction in Younger and Older Women. J Woman Aging. 2006; 18: 41-55

25. Banfield S, McCabe R. An evaluation of the construct of body image. Adolescence. 2002; 37: 373-393.

26. Tiggemann M. Body image across the adult life span: stability and change. Body Image. 2004; 1: 29-41.

27. Runfola C, Holle A, Trace S, Brownley K, Hofmeier $\mathrm{S}$, Gagne D et al. Body Dissatisfaction in women across the lifespan: results of the UNC-SELF and gender and Body Image (GABI) Studies. Eur Eat Disord Rev. 2012; 21: 52-59

28. Tiggemann M, Lynch J. Body image across the life 
span in adult women: The role of self-objectification. Dev Psychol. 2001; 37: 243-253.

29. Camacho J, Fernández E. Imagen corporal y práctica de actividad física en las chicas adolescentes: incidencia de la modalidad deportiva. Int J Sport Sci. 2006; 2: 1-19.

30. Williams PA, Cash TF. Effect of a circuit weight training program on the body images of college students. Int J Eat Disord. 2001; 30, 75-82.

31. Smolak L, Murner S, Ruble AE. Female athletes and eating problems: a meta-analysis. Int J Eat Disord. 2009; 27: 371-80

32. Phelps L, Jennifer S, Nathanson, D, Nelson L. An empirically supported eating disorder prevention program. Psychol sch. 2000; 37: 443-452.

33. Tiggemann M. The impact of adolescent girls' life concerns and leisure activities on body dissatisfaction, disordered eating and self-esteem. J Genet Psychol. 2001; 162: 133-142.
34. Powers PS, Johnson C. Small victories: prevention of eating disorders among athletes. Eat disord. 1996; 4: 364-367.

35. Mäkinen M, Puukko-Viertomies L, Lindberg N, Siimes M. Body dissatisfaction and body mass in girls and boys transitioning from early to mid-adolescence: additional role of self-esteem and eating habits. BMC Psychiatry. 2012; 12: 35.

36. Cheung Y, Lee A, Yin S, Shing E, Lam T. Who wants a slimmer body? The relationship between body weight status, education level and body shape dissatisfaction among young adults in Hong Kong. BMC Public Health. 2011; 11: 835.

37. Neighbors L, Sobal J. Prevalence and magnitude of body weight and shape dissatisfaction among university students. Eat Behav. 2007; 8: 429-439

38. Ferreiro F, Seoane G, Senra C. A prospective study of risk factors for the development of depression and disordered eating in adolescents. J Clin Child and Adolesc Psychol. 2011; 40: 500-505. 